\title{
An Oligonucleotide Probe Derived from kDNA Minirepeats is Specific for Leishmania (Viannia)
}

\section{Octavio Fernandes/*/+, Marcelo Bozza**, Juan M Pascale***, Antonio B de Miranda, Ulisses G Lopes**, Wim M Degrave}

Departamento de Bioquímica e Biologia Molecular, Instituto Oswaldo Cruz, Av. Brasil 4365, 21045-900 Rio de Janeiro, RJ, Brasil *Departamento de Patologia, Faculdade de Ciências Médicas, Universidade do Estado do Rio de Janeiro, RJ, Brasil **Departamento de Biofísica, Universidade Federal do Rio de Janeiro, RJ, Brasil ***Departamento de Parasitología, Universidad de Panama, Panama

Sequence analysis of Leishmania (Viannia) kDNA minicircles and analysis of multiple sequence alignments of the conserved region (minirepeats) of five distinct minicircles from $\mathrm{L}$. (V.) braziliensis species with corresponding sequences derived from other dermotropic leishmanias indicated the presence of a sub-genus specific sequence. An oligonucleotide bearing this sequence was designed and used as a molecular probe, being able to recognize solely the sub-genus Viannia species in hybridization experiments. A dendrogram reflecting the homologies among the minirepeat sequences was constructed. Sequence clustering was obtained corresponding to the traditional classification based on similarity of biochemical, biological and parasitological characteristics of these Leishmania species, distinguishing the Old World dermotropic leishmanias, the New World dermotropic leishmanias of the sub-genus Leishmania and of the sub-genus Viannia.

Key words: Leishmania (Viannia) identification - minicircle DNA sequences - oligonucleotide probe Leishmania braziliensis - Leishmania panamensis

The parasitological diagnosis of leishmaniasis has received, in recent years, a growing attention due to the development of molecular probes, capable both of detecting and typing the infecting parasite (Ramirez \& Guevara 1987, Van Eys et al. 1987, 1991, Howard et al. 1991, Degrave et al. 1994a, b). Traditional methods for identification are slow and cumbersome, and suffer from a lack of sensitivity (Weigle et al. 1987). Thus, methods like culture of the protozoa from human samples, microscopic analysis of stained smears or histopathological study of biopsy material might be replaced in the future by fast, sensitive and accurate diagnostic assays, using molecular probes and/or the polymerase chain reaction (PCR)(Barker et al. 1986, Barker 1987, Uliana et al. 1991).

Probes derived from different sequences of the nuclear and mitochondrial genome have been used in an attempt to develop Leishmania detection and typing assays (Wirth \& McMahon-Pratt 1982, Van Eys et al. 1992). However, the kinetoplast DNA

Sponsored by the UNDP/World Bank/WHO Special Program for Research and Training in Tropical Diseases (TDR), the International Atomic Energy Agency, the FIOCRUZ PAPES Program and CNPq.

${ }^{+}$Corresponding author. Fax: 55-21-590.3495

Received 8 February 1995

Accepted 1 February 1996
(kDNA) network, which is comprised of maxi- and minicircle molecules, has been the target of choice in many laboratories (Simpson 1987). Leishmania minicircles show features that make them almost ideal as molecular probes, since they are present in the kinetoplast network in a high copy number and contain a conserved region of at least $120 \mathrm{bp}$ that can be evidenced in every molecule (Rogers \& Wirth 1987, 1988). Specific probes based on total kDNA, complete minicircle molecules or restriction fragments, have been generated and used in the identification of different leishmanias from the Old and New World (Lopes \& Wirth 1986, Das Gupta et al. 1991, Laskay et al. 1991). This approach proved to be suitable for epidemiological purposes and as a diagnostic method (Wirth et al. 1986, Rogers et al. 1988, Barker 1989).

Detection systems based on PCR are very promising due to their sensitivity and specificity (Saiki et al. 1985). However, secondary detection and specific typing probes for identification of PCR amplified products are highly desirable, since they offer a drastically increased specificity of the PCR assay and raise about ten-fold the sensitivity of the system, allowing for automation of the PCR scoring procedure, concomitantly with the typing of the infecting Leishmania species. Several PCR systems use amplification oligonucleotides complementary to the conserved sequence motifs corresponding to the origins of replication of the 
minicircle light and heavy strands (Rodgers et al. 1990).

Whole kDNA can be used as a molecular probe to distinguish between the two New World dermotropic Leishmania groups: the sub-genus Viannia (braziliensis, guyanensis, panamensis, etc.) and Leishmania (amazonensis, mexicana, etc.) (Wirth \& McMahon-Pratt 1982). However, cross hybridization with Old World leishmanias and $L$. chagasi occurs (Das Gupta et al. 1991). Synthetic oligonucleotides directed to specific sequences can circumvent these problems and can be used in a PCR assay.

We report here the partial nucleotide sequences of L. braziliensis and L. panamensis minicircles and the identification of an oligonucleotide sequence specific to the subgenus Viannia, based on multiple sequence alignments of the minicircles conserved regions (minirepeats) from New World dermotropic leishmanias. Furthermore, a dendrogram reflecting the different minirepeat sequence homologies between these dermotropic leishmanias was constructed.

\section{MATERIALS AND METHODS}

Parasite culture and kDNA isolation - The Leishmania strains used in this study were: $L$. braziliensis $\mathrm{MHOM} / \mathrm{BR} / 75 / \mathrm{M} 2903$, kindly provided by Dr Gabriel Grimaldi, FIOCRUZ, Rio de Janeiro, and $L$. panamensis IPAN V (Panama University, Panama). Promastigotes were grown at $24^{\circ} \mathrm{C}$ in Schneider's medium (GIBCO, Grand Island, NY) supplemented with $10 \%$ heat-inactivated fetal calf serum. The taxonomic nomenclature follows the recommendation of the World Health Organization.

The cells were spun down at $5000 \mathrm{Xg}$ for 10 $\min$ at $4^{\circ} \mathrm{C}$, washed with cold saline/EDTA $(0,15$ $\mathrm{M} \mathrm{NaCl} / 10 \mathrm{mM}$ EDTA), centrifuged and resuspended in $\mathrm{TE}_{10}(10 \mathrm{mM}$ Tris- $\mathrm{HCl} \mathrm{pH} 7.4 / 10 \mathrm{mM}$ EDTA). Proteinase K (Boehringer Mannheim) and sarkosyl were added to final concentrations of 100 $\mu \mathrm{g} / \mathrm{ml}$ and $0.5 \%$, respectively. The lysate was incubated at $56^{\circ} \mathrm{C}$ for $2 \mathrm{hr}$ and submitted to mechanical shear, followed by two ultracentrifugations at $30.000 \mathrm{rpm}$ for $1 \mathrm{hr}$ at $4^{\circ} \mathrm{C}$. The pellet was resuspended in $200 \mu \mathrm{l}$ of TE buffer and extracted with phenol/chloroform (1:1). The DNA was precipitated with ethanol and resuspended in $\mathrm{TE}_{1}(10 \mathrm{mM}$ Tris-HCl pH 7.4/ 1 mM EDTA).

Minicircle cloning and nucleotide sequence determination - Total L. braziliensis kDNA was digested with Msp I and minicircles were cloned in the Acc I site of $\mathrm{M}_{13} \mathrm{mp} 18$. L. panamensis was digested with Dra I and cloned in the Hinc II site of pBluescript ${ }^{\circledR}$ KS-. Nucleotide sequencing was done by the chain termination method using either
$\mathrm{USB}^{\circledR}$ Sequenase kit and $\alpha-{ }^{32} \mathrm{P}-\mathrm{dATP}$ labeling or the Taq Dye Primer Cycle Sequencing Kit on a 373A Automated DNA Sequencer (Applied Biosystems), according to the instructions of the manufacturer.

Computer analysis and oligonucleotide synthesis - Nucleotide sequences were analyzed using software from the Genetics Computer Group (GCG) on a VAX computer. Oligonucleotides were synthesized on an Applied Biosystems 381A DNA synthesizer and purified by thin layer chromatography (TLC).

Cell dot experiments and hybridization assays - Leishmania cells were pelleted, washed with $\mathrm{TE}_{10}$ and counted. $10^{5}$ cells were applied on nylon filters (Zetaprobe, Biorad) treated with $10 \mathrm{mM}$ Tris$\mathrm{HCl} \mathrm{pH}$ 8.0, $10 \mathrm{mM}$ EDTA, 0.1\% Triton X-100, $100 \mu \mathrm{g} / \mathrm{ml}$ proteinase $\mathrm{K}$ at $56^{\circ} \mathrm{C}$ during $1 \mathrm{hr}$. The filter was transferred to $0.4 \mathrm{~N} \mathrm{NaOH}$ for $5 \mathrm{~min}$ and then rinsed with 2xSSC and dried. Hybridization conditions and washing stringencies are detailed below in results.

\section{RESULTS}

Minicircle cloning and sequencing - One $L$. panamensis, one L. amazonensis and several cloned L. braziliensis minicircles were partially sequenced and the data were submitted to GenBank (Fig. 2, Table). The molecules apparently belong to different minicircle classes, as judged from the extensive heterogeneity of the minirepeat neighboring sequences (data not shown).

Multiple sequence alignment of minirepeats We performed a multiple alignment analysis of the conserved minirepeats from New and Old World dermotropic Leishmania minicircles (Fig. 2), using the Pileup program from the GCG package. Regions with higher or lower conservation become evident when comparing the alignment with a deduced consensus sequence. A dendrogram reflecting the homologies between these sequences is shown in Fig. 3. Although this dendrogram does not necessarily reflect a phylogenetic relationship between the different Leishmania species, it does cluster the Leishmania species in groups that are coherent with previous classifications based upon biological, parasitological and biochemical observations (WHO 1980).

Oligonucleotide design - The multiple alignment of the minirepeats regions has enabled us to evidence a region, adjacent to the 5 ' end of the canonical twelvemer sequence, that seems highly specific to leishmanias of the sub-genus Viannia. An oligonucleotide (5'-TAATTGTGCACGGGGA GGCCA-3' - OLB) corresponding to this region was synthesized and used in hybridization experiments as a probe, confirming the specificity sug- 


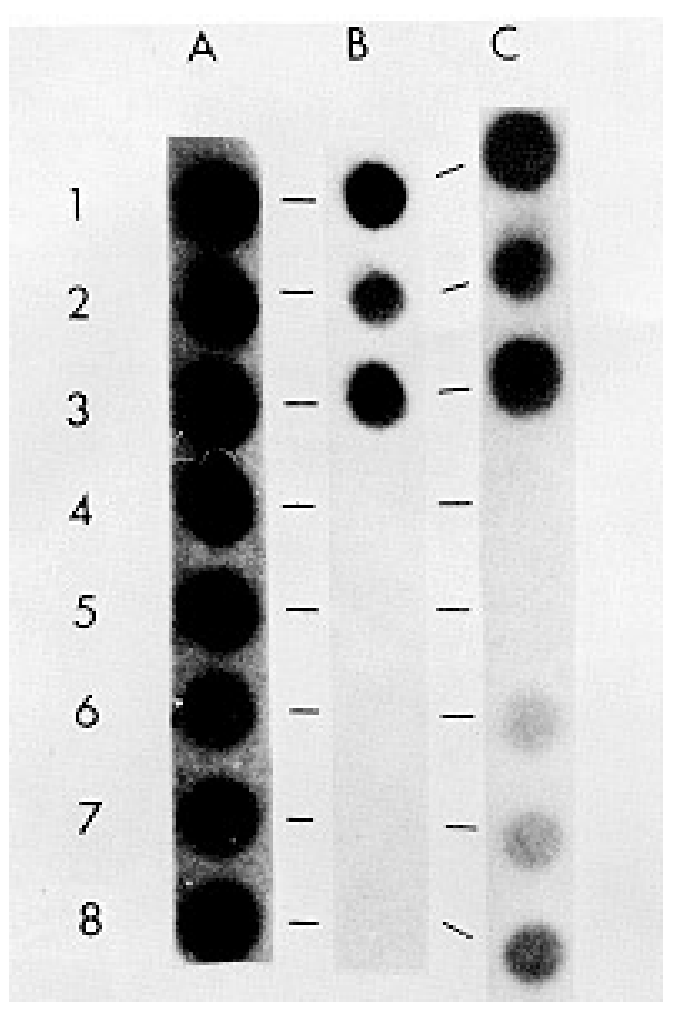

Fig. 1: cell dot hybridization of different Leishmania species with the oligonucleotide OLB (1.A), OLT (1.B) or $L$. braziliensis total kDNA (1.C) as molecular probes. 1) L. (V.) guyanensis (M4147); 2) L. (V.) braziliensis (M2309); 3) L. (V.) panamensis (IPAN5); 4) L. (L.) amazonensis (M2269); 5) L. (L.) major (JERICHO II); 6) L. (L.) tropica (OD); 7) L. (L.) chagasi (BA3) and 8) L. (L.) infantum (IPTI). gested by the sequence analysis. Using stringent hybridization conditions and washing in $0.5 \mathrm{xSSC}$ at $37^{\circ} \mathrm{C}$, we demonstrated that this oligonucleotide is specific to the sub-genus Viannia (Fig. 1A), in contrast to hybridization with an oligonucleotide complementary to the minicircle "universal 12-mer sequence" (5'-GGGGTTGGTGTAAT/AATAGG/ TGGC-3'- OLT) (Fig. 1B) or with L. brasiliensis total kDNA (Fig. 1C).

\section{DISCUSSION}

Leishmania species belonging to the sub-genus Viannia are the main agents of cutaneous leishmaniasis in Latin America. However, there are other leishmanias from the sub-genus Leishmania, which are also responsible for human cutaneous infections (Grimaldi \& Tesh 1993). In some areas there is an overlapping of different dermotropic or viscerotropic leishmanias, creating a complex epidemiologic pattern of transmission. Moreover, the prognosis of human leishmaniasis seems also to depend on the species involved in the infection. It is thus highly desirable to develop molecular probes that are able to identify and type the different leishmanias (Barker 1989).

Since the use of oligonucleotides as molecular probes is inexpensive, more specific, faster and more practical as compared to whole kDNA, we cloned and partially sequenced five different minicircles from $L$. $(V$.) braziliensis, searching for short sequences specific to minicircles from this relevant human pathogenic parasite group.

TABLE

Minicircle sequences used in the multiple alignment

\begin{tabular}{|c|c|c|c|}
\hline $\begin{array}{l}\text { Minicircle } \\
\text { nomenclature }\end{array}$ & Leishmania strain & $\begin{array}{c}\text { GenBank } \\
\text { accession } \\
\text { number }\end{array}$ & Described by \\
\hline L. braziliensis 1 & MHOM/BR/75/2903 & U19803 & this article \\
\hline L. braziliensis 2 & MHOM/BR/75/2903 & U19804 & this article \\
\hline L. braziliensis 3 & MHOM/BR/75/2903 & U19805 & this article \\
\hline L. braziliensis 4 & MHOM/BR/75/2903 & U19806 & this article \\
\hline L. braziliensis 5 & MHOM/BR/75/2903 & U19807 & this article \\
\hline L. braziliensis 6 & MHOM/BR/75/2904 & M87315 & de Bruijin \& Barker 1992 \\
\hline L. guyanensis 1 & $151 \mathrm{M}$ & X54470 & Samuelson et al. 1991 \\
\hline L. guyanensis 2 & $151 \mathrm{M}$ & X54471 & Samuelson et al. 1991 \\
\hline L. guyanensis 3 & $151 \mathrm{M}$ & X54472 & Samuelson et al. 1991 \\
\hline L. guyanensis 4 & UMB/BR/76/M4196 & M87316 & de Bruijin \& Barker 1992 \\
\hline L. panamensis 1 & MHOM/PA/75/M4037 & M87314 & de Bruijin \& Barker 1992 \\
\hline L. panamensis 2 & IPAN V & U19811 & this article \\
\hline L. peruviana & MHOM/PE/76/SL5 & M87317 & de Bruijin \& Barker 1992 \\
\hline L. amazonensis 1 & IFLA/BR/67/PH8 & & Rogers \& Wirth 1987 \\
\hline L. amazonensis 2 & MHOM/BR/73/M2269 & U19810 & this article \\
\hline L. major & MHOM/TN/84/ISS108 & J04654 & Smith et al. 1989 \\
\hline L. aethiopica & pPK763 & & Barker et al. 1986 \\
\hline L. tarentolae & Clonal strain $\mathrm{C}-1$ & K01979 & Kidane et al. 1984 \\
\hline
\end{tabular}




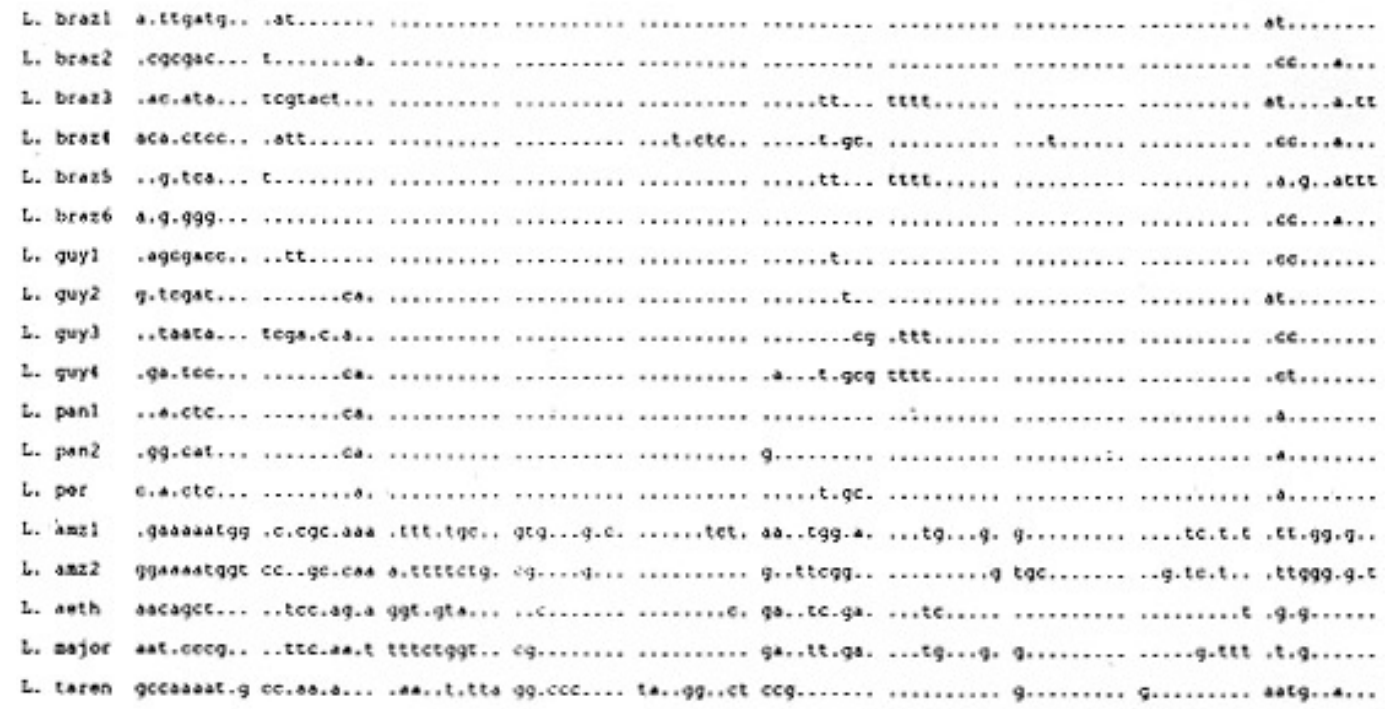

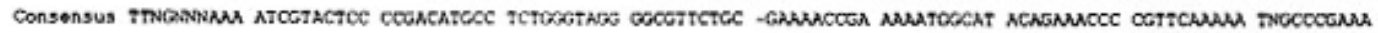

10 :

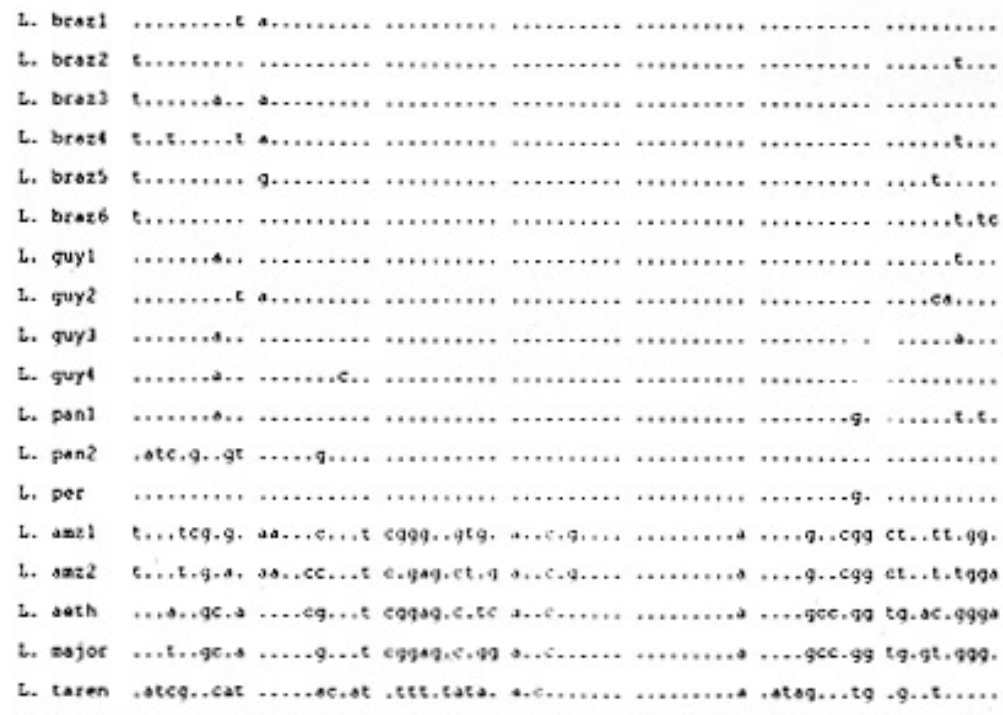

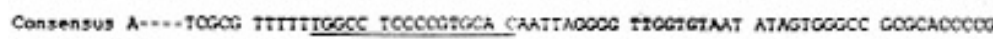

Fig. 2: multiple alignment of $170 \mathrm{bp}$ of the conserved region from six different minicircle molecules of Leishmania braziliensis, four minicircles of $L$. guyanensis, two of $L$. panamensis, two of $L$. amazonensis, one of $L$. peruviana, L. aethiopica, L. major and L. tarentolae. The leishmania strains, the minicircle nomenclature and the GenBank accession numbers are listed in Table. The conserved 12mer sequence, present in all Leishmania minicircles, is marked in bold. The Viannia specific oligonucleotide sequence is underlined. Nucleotide positions identical to the consensus sequence were marked with a dot.

Based on the multiple alignment of the obtained sequences with those of the conserved regions of other Leishmania minicircles from different species, we identified a region of 16 nucleotides (OLB, Fig. 2), that proved to be specific for the sub-ge- nus Viannia in cell dot hybridization experiments (Fig. 1), demonstrating its use as a typing probe. This region is $5^{\prime}$ adjacent to the conserved block of 12 nucleotides, found in all Leishmania minicircles, for which another oligonucleotide was 


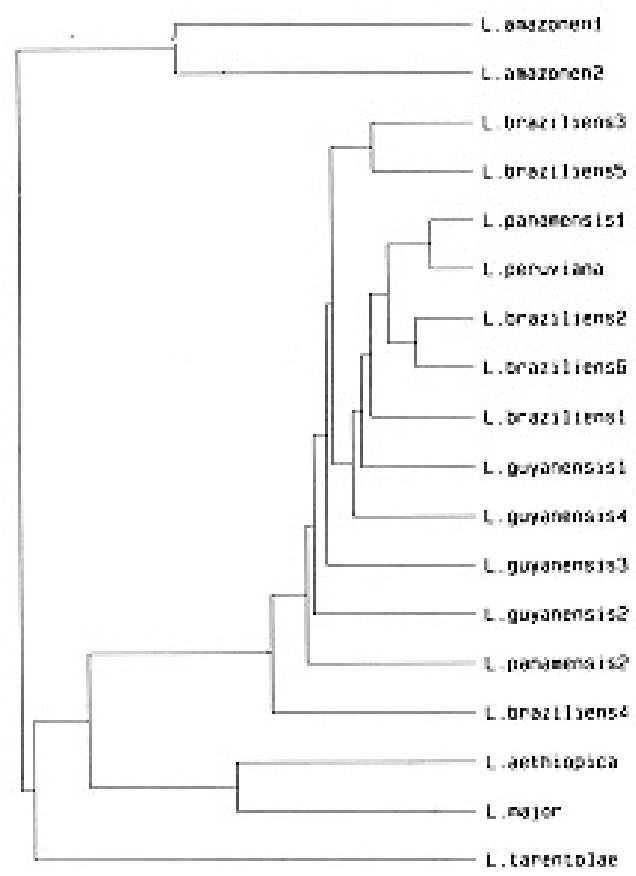

Fig. 3: dendrogram derived from the Pileup (GCG package) analysis, using the conserved region of dermotropic leishmanias. The minicircle nomenclature is listed in Table.

synthesized (OLT, Fig. 1B, Fig. 2). Apart from its use as a typing probe in cell-dot experiments, the OLB oligoprobe proved to be very useful for the detection and typing of PCR products (Degrave et al. 1994a), generated using oligonucleotides complementary to the minicircle light and heavy strand origins of replication.

Moreover, using oligonucleotides directed outwardly from the Viannia specific region and from the origin of replication of the minicircle heavy strand (12mer sequence), as primers in a PCR reaction, it is possible to amplify the whole minicircle molecule. This method may be useful in typing experiments in a schizodeme-like analysis after full length minicircle amplification (Degrave et al. 1994b). De Bruijn and Barker (1992) have previously used such amplification of whole minicircles for diagnostic purposes.

The L. Viannia minirepeat sequences demonstrate extensive sequence conservation. Moreover, point mutations are clustered in a few regions and do not distinguish between the different species, as shown by the clustering produced by the computer analysis of the Leishmania minirepeats. All the Viannia species are grouped together, with a near homogeneity inside the sub-genus taxon in Leishmania species when considering the con- served region of the minicircle molecule. Species of the sub-genus Leishmania are also grouped, and separated from the Viannia cluster.

\section{ACKNOWLEDGEMENTS}

To Dr Gabriel Grimaldi for strains donations and culture facilities.

\section{REFERENCES}

Barker D 1987. DNA Diagnosis of Human Leishmaniasis. Parasitol Today 3: 177-184.

Barker D 1989. Molecular approaches to DNA diagnosis. Parasitol 99: 129-146.

Barker DC, Gibson LJ, Kennedy WPK, Nasser AAAA, Williams RH 1986. The potential of using recombinant DNA species-specific probes for the identification of tropical Leishmania. Parasitol 91: S139S174.

Das Gupta S, Ghosh DK, Majumder HK 1991. A cloned kinetoplast DNA minicircle fragment from a Leishmania sp. specific for post-kala-azar dermal leishmaniasis strains. Parasitol 102: 187-191.

De Bruijn M, Barker D 1992. Diagnosis of New World leishmaniasis: specific detection of species of the Leishmania braziliensis complex by amplification of kinetoplast DNA. Acta Tropica 52: 45-48.

Degrave WM, Fernandes O, Campbell D, Bozza M, Lopes UG 1994b. Use of molecular probes and PCR for detection and typing of Leishmania - A minireview. Mem Inst Oswaldo Cruz 89: 463-469.

Degrave WM, Fernandes O, Thiemann O, Wincker P, Britto C, Cardoso A, Pereira JB, Bozza M, Lopes U, Morel C 1994a. Detection of Trypanosoma cruzi and Leishmania using the Polymerase Chain Reaction. Mem Inst Oswaldo Cruz 89: 367-368.

Grimaldi G, Tesh RB 1993. Leishmaniases of the New World: current concepts and implications for future research. Clin Microbiol Rev 6: 230-250.

Howard MK, Kelly JM, Lane RP, Miles MA 1991. A sensitive repetitive DNA probe that is specific to the Leishmania donovani complex and its use as an epidemiological and diagnostic reagent. Mol Biochem Parasitol 44: 63-72.

Kidane G, Hughes D, Simpson L 1984. Sequence heterogeneity and anomalous electrophoretic mobility of kinetoplast minicircle DNA in Leishmania tarentolae. Gene 27: 265-277.

Laskay T, Kiessling R, Dewit TFR, Wirth DF 1991. Generation of species-specific DNA probes for Leishmania aethiopica. Mol Biochem Parasitol 44: 279286.

Lopes UG, Wirth DF 1986. Identification of visceral Leishmania species with cloned sequences of kinetoplast DNA. Mol Biochem Parasitol 20: 77-84.

Ramirez JL, Guevara P 1987. The ribosomal gene spacer as a tool for the taxonomy of Leishmania. Mol Biochem Parasitol 22: 177-183.

Rodgers MR, Popper SJ, Wirth DF 1990. Amplification of kinetoplast DNA as a tool in the detection and diagnosis of Leishmania. Exp Parasitol 71: 267275.

Rogers WO, Wirth DF 1987. Generation of sequence 
diversity in the kinetoplast DNA minicircles of Leishmania mexicana amazonensis. Mol Biochem Parasitol 30: 1-8.

Rogers WO, Burnheim PF, Wirth DF 1988. Detection of Leishmania within sand flies by kinetoplast DNA hybridization. Am J Trop Med Hyg 39: 434-439.

Saiki R, Scharf SJ, Faloona F, Mullis K, Horn G, Erlich H, Arnheim N 1985. Enzymatic amplification of beta globin genomic sequence and restriction site analysis for diagnosis of sickle cell anemia. Science 230: 1350-1354.

Samuelson J, Lerner E, Tesh R, Titus R 1991. A mouse model of Leishmania brasiliensis brasiliensis infection produced by coinjection with sandfly saliva. $J$ Exp Med 173: 49-54.

Simpson L 1987. The mitochondrial genome of kinetoplastid protozoa: genomic organization, transcription, replication and evolution. Annu Rev Microbiol 41: 363-382.

Uliana SRB, Affonso MHT, Camargo EP, Floeter-Winter LM 1991. Leishmania: genus identification based on a specific sequence of the $18 \mathrm{~S}$ ribosomal RNA sequence. Exp Parasitol 72: 157-163.

Van Eys GJJM, Guizani I, Ligthart GS, Dellagi K 1991. A nuclear DNA probe for the identification of strains within the Leishmania donovani complex. Exp Parasitol 72: 459-463.
Van Eys GJJM, Schoone GJ, Kroon NCM, Ebeling SB 1992. Sequence analysis of small subunit ribosomal RNA genes and its use for detection and identification of Leishmania parasites. Mol Biochem Parasitol 51: 133-142.

Van Eys GJJM, Schoone GJ, Ligthart GS, Laarman JJ, Terpstra WJ 1987. Detection of Leishmania parasites by DNA in situ hybridization with non-radioactive probes. Parasitol Res 73: 199-202.

Weigle KA, Davalos M, Heredia P, Molineros R, Saravia N, D'Alessandro A 1987. Diagnosis of cutaneous and mucocutaneous leishmaniasis in Colombia: a comparison of seven methods. Am J Trop Med Hyg 36: 489-496.

WHO 1980 - World Health Organization - Biochemical characterization of Leishmania. Proceedings of a workshop held at the Pan American Health Organization, Washington, D.C. ML Chance \& BC Walton (eds).

Wirth DF, McMahon-Pratt D 1982. Rapid identification of Leishmania species by specific hybridization of kinetoplast DNA in cutaneous lesions. Proc Nat Acad Sci USA 77: 6810-6814.

Wirth DF, Rogers WO, Barker R, Dourado H, Suesebang L, Albuquerque B 1986. Leishmaniasis and malaria: new tools for epidemiologic analysis. Science 234 : 975-979. 